\section{Setting straight the record}

\author{
EDWARD C. CARTERETTE \\ University of California, Los Angeles \\ Los Angeles, California \\ and \\ NORMAN H. ANDERSON \\ University of California, San Diego \\ La Jolla, California
}

The monotone analysis used by Carterette and Anderson (1979) is misrepresented by Birnbaum (1982). Birnbaum attributes to us a procedure that we did not use, but that we severely criticized. Birnbaum then presents an alternative approach like the one we actually did use.

The issue concerns monotone transformation to additivity. Monotone transformation is so flexible that it may make nonadditive data falsely appear additive. We pointed out that the common practice of using monotone transformation with single replications did not protect against the danger of such inappropriate transformation. Single replication refers to a single score in each design cell, even thought this single score may be the mean or median of several scores. Instead, we used a distribution method, based on the ADDALS program of de Leeuw, Young, and Takane (1976), in which several replications are transformed with a single transformation. We cited evidence to show that this distribution method could provide additional constraint to prevent false transformation to additivity.

Our procedure was clearly specified, as the following quotes from Carterette and Anderson (1979) indicate.

[Monotone transformation] was applied separately to the first five and to the second five replications.... Two independent estimates of the best monotone transformation were thus obtained for each subject for each task. (p. 275)

[This procedure] has the particular advantage of transforming the entire distribution of responses in each cell rather than only their mean. (p. 275)

It seems quite clear that a single score in each cell of the design does not ordinarily provide adequate constraint. (p. 276)

One replication per cell does not provide adequate power. (p. 276)

The above results indicate that monotone analysis is far more demanding than has been recognized.... However, the procedure of transforming a number of replications simultaneously does show provisional promise to provide adequate power for monotone analysis. (p. 277)

Address correspondence to E. C. Carterette, Perceptual Systems Laboratory, Department of Psychology, University of California, Los Angeles, CA 90024.
As these quotes illustrate, we explicitly emphasized that monotone transformation based on a single replication was inadequate, a conclusion for which we presented detailed evidence. We also presented evidence to indicate provisional promise for our distribution method of transforming several replications at a time.

Birnbaum (1982), however, repeatedly makes the factually incorrect assertion that we transformed single replications. Some of these misstatements appear in the following quotes from his note.

Their procedure employs a separate transformation for each replicate. (p. 293)

Monotone functions, estimated separately for each replicate. (p. 293)

However, when different monotonic transformations are permitted for each replicate. (p. 294)

Carterette and Anderson's (1979) procedure, which uses different transformations for different replicates. (p. 294)

[Their] procedure does not truly use the distribution of responses as a constraint for transformation. (p. 295)

Birnbaum then goes on to advocate using the distribution of responses as a constraint for transformation by transforming several replications at a time. Birnbaum thus attributes to us a single replication procedure, which we explicitly rejected, and advocates a distribution procedure, which is what we used.

Birnbaum's discussion of scale-free tests is similarly misleading. Scale-free, crossover tests were used extensively in information integration theory beginning in 1965, long before Birnbaum employed them (see Anderson, 1982, Notes $5.4 \mathrm{~h}$ and $7.1 \mathrm{~d}$ ).) In a different sense, functional measurement is in principle scale-free, for it bases measurement solely on the structure of the integration function. This scale-free characteristic is well illustrated in our bisection study:

No auxiliary assumptions are necessary.... All that is at issue is the algebraic form of the bisection model. This algebraic form serves as the base and frame for measurement in a truly scale-free way. (p. 266)

Monotone analysis still presents serious difficulties. We understand Birnbaum's concern over the pitfalls of monotone analysis since so much of his own previous work rests on this technique. Our original article showed that a distribution method could provide useful constraint to prevent inappropriate monotone transformation, although we noted that the statistical properties of the distribution method required study. We also noted the special value of two-operation models to bypass the main problem with monotone analysis (see Anderson, 1982, chap. 5). These statistical issues, however, are outside the scope of this comment. Our concern here is merely to set straight the 
record: We did not use the single replication method, as Birnbaum claimed; we did use a distribution method, as Birnbaum later advocated.

\section{REFERENCES}

ANDERSON, N. H. (1982). Methods of information integration theory. New York: Academic Press.

Birnbaum, M. H. (1982). On rescaling data to fit the model and con- cluding that the model fits: A note on monotone transformation. Perception \& Psychophysics, 32, 293-296.

Carterette, E. C., \& ANDerson, N. H. (1979). Bisection of loudness. Perception \& Psychophysics, 26, 265-280.

De LeEuw, J., Young, F. W., \& TAKane, Y. (1976). Additive structure in qualitative data: An alternating least squares method with optimal scaling features. Psychometrika, 41, 471-503.

(Manuscript received April 20, 1987; accepted for publication April 25, 1987.)

\section{ERRATUM}

Skowbo, D., \& Forster, T. (1983). Further evidence against the classical conditioning model of McCollough effects. Perception \& Psychophysics, 34, 552-554.

Skowbo, D., Garrity, S., \& Michaud, L. (1985). Does testing initiate decay of McCollough effects? Perception \& Psychophysics, 37, 377-381.

Skowbo, D., \& Michaud, L. (1985). Individual differences in McCollough effects: A study of twins. Perception \& Psychophysics, 38, 208-210.

An error in luminance conversions was recently discovered in the original records of these experiments. Correct luminance values in $\mathrm{cd} / \mathrm{m}^{2}$ may be obtained by multiplying reported values by 11.737 . 